\title{
Functional properties of a newly cloned fish ortholog of the neutral amino acid transporter $\mathrm{B}^{0} \mathrm{AT} 1$ (SLC6A19)
}

\author{
Eleonora Margheritis ${ }^{\mathrm{a}, \mathrm{b}}$, Genciana Terova ${ }^{\mathrm{a}}$, Raffaella Cinquetti ${ }^{\mathrm{a}}$, Antonio Peres ${ }^{\text {a }}$, Elena Bossi ${ }^{\mathrm{a}, *}$ \\ a Department of Biotechnology and Life Sciences, University of Insubria, Via J.H. Dunant 3 I-21100, Varese, Italy \\ b School of Biological and Medical Sciences, University of Insubria, Varese, Italy
}

\section{A R T I C L E I N F O}

Article history:

Received 24 April 2013

Received in revised form 20 June 2013

Accepted 26 June 2013

Available online 1 July 2013

\section{Keywords:}

Nutrient transport

SLC6A19

$\mathrm{B}^{0} \mathrm{AT} 1$

Neutral amino acid transporter

Essential amino acid (EAA)

Xenopus oocytes

\begin{abstract}
A B S T R A C T
The functional properties of an ortholog of the $\mathrm{B}^{0} \mathrm{AT} 1$ (SLC6A19) amino acid transporter, cloned from the intestine of the sea bass Dicentrachus labrax, were investigated. The two-electrode voltage-clamp technique was applied to Xenopus laevis oocytes heterologously expressing the transporter in order to measure the currents associated with the transport process in different conditions. In particular the substrate specificity, the ionic requirements, and possible effects of $\mathrm{pH}$ were examined. Among the organic substrates, leucine, glycine, serine and valine generated the largest transport currents with apparent affinities in the lower millimolar range. The importance of $\mathrm{Na}^{+}$as the driver ion in the transport process is confirmed, although $\mathrm{Li}^{+}$is also capable to sustain transport, while $\mathrm{K}^{+}$is not. No evidence of a relevant role of $\mathrm{Cl}^{-}$in the transport activity was found. Concerning the other two kinds of currents commonly found in electrogenic transporters, very fast pre-steady-state currents were detected in the absence of organic substrate, while lithium-specific leak currents were not observed. The comparison of these properties with those of the mammalian and insect orthologs may give interesting indication for future structure-function studies in this transporter subfamily.
\end{abstract}

(c) 2013 Elsevier Inc. All rights reserved.

\section{Introduction}

Absorption of amino acids in the animal intestinal tract and reabsorption in the kidney are accomplished by specific transport proteins located in the apical and basolateral membranes of the appropriate epithelial cells. Uphill intake of amino acids from the intestinal lumen is permitted by secondary active cotransporters in the apical membrane, such as the proton-coupled di- and tri-peptide transporter PepT1 (SLC15A1) (Daniel and Kottra, 2004; Bossi et al., 2011; Renna et al., 2011), and the $\mathrm{Na}^{+}$- coupled neutral amino acid transporter $\mathrm{B}^{0}$ AT1 (SLC6A19) (Böhmer et al., 2005; Camargo et al., 2008). While PepT1 has been cloned and characterized in vertebrates and invertebrates, including bacteria and yeast (Daniel et al., 2006), the functional characterization of $\mathrm{B}^{0} \mathrm{AT} 1$ is limited to mammalian orthologs (Kleta et al., 2004; Böhmer et al., 2005; Camargo et al., 2005) and to some insect transporters classified as $\mathrm{B}^{0} \mathrm{AT} 1$-like, such as msKAAT1, msCAATCH1 and dmNAT1 (Bossi et al., 1999a; Feldman et al., 2000; Boudko et al., 2005; Bossi et al., 2007b; Miller et al., 2008).

The understanding of the functional properties of transporters responsible for nutrient absorption is obviously relevant under various points of view, such as the comprehension and modelling of the molecular transport mechanisms (Boudko, 2012), the assessment of their implication in pathological conditions (Seow et al., 2004; Bröer et al.,

\footnotetext{
* Corresponding author. Tel.: + 390332421318 ; fax: + 3903321421300 E-mail address: elena.bossi@uninsubria.it (E. Bossi).
}

2005), or the establishment of optimal diet composition in animal farming (Edwards et al., 1999; Verri et al., 2011; Kwasek et al., 2012; Margheritis et al., 2012).

In connection with this last aspect, recently a fish ortholog from the sea bass Dicentrachus labrax, has been cloned ( $\mathrm{sbB}^{0} \mathrm{AT} 1$ ) (GenBank KC812315, Terova, in preparation).

In the present work we have studied the functional properties of $\mathrm{sbB}^{0} \mathrm{AT} 1$ using electrophysiological methods in Xenopus laevis oocytes heterologously expressing the protein. The amino acid specificity, the ionic selectivity and the apparent affinity of the transporter were characterized by analyzing the transport associated current. Furthermore, we investigated the presence of two other kinds of current frequently observed in ion coupled cotransporters, namely the transmembrane "leak" or "slippage" current (Nelson et al., 2002; Andrini et al., 2008), and the intramembrane pre-steady-state current caused by the rearrangement of the transport protein and/or to the displacement of mobile ions in the membrane electric field (Hazama et al., 1997; Peres et al., 2004). These properties were compared to those of the mammalian and insect orthologs to highlight similarities and differences that could be useful for structure function studies.

\section{Materials and methods}

\subsection{Oocyte preparation and $m R N A$ injection}

Xenopus laevis oocytes and RNAs were prepared as previously described in detail (Bossi et al., 2007a). The cDNA encoding for 
$\mathrm{sbB}^{0} \mathrm{AT} 1$ was cloned from the gut of Dicentrarchus labrax (GenBank AN. KC812315; Terova, in preparation) and it was inserted into the pSPORT-1 vector as the cDNA coding for msKAAT1 (Invitrogen, Milan, Italy, www.lifetechnologies.com). The cDNA of mouse collectrin kindly provided by Prof. Joseph Penninger (IMBA Institute of Molecular Biotechnology Vienna, Austria) was in pCDNA3.1(Camargo et al., 2008), the mouse $\mathrm{B}^{0} \mathrm{AT} 1$, kindly provided by Simone Camargo (Institute of Physiology, University of Zurich) was into a pBlueScript modified Xenopus expression vector (KSM). For the expression in Xenopus oocytes the mouse $\mathrm{B}^{0} \mathrm{AT} 1-\mathrm{KSM}$ plasmid was linearized with XbaI (Promega) and used as template for RNA synthesis from the T3 promoter. The other plasmids were linearized with NotI for $\operatorname{sbB}^{0} \mathrm{AT} 1$ and msKAAT1 and with Smal for mouse collectrin and synthesized from the T7 promoter, All cRNAs were transcribed in vitro in the presence of Cap Analog and 200 units of specific RNA polymerase. All enzymes were supplied by Promega Italia (www. promega.com, Milan, Italy). Oocytes were obtained from adult female $X$. laevis (Xenopus express, France, www.xenopus.com); the frogs were anesthetized in MS222 (tricaine methanesulfonate salt) (Sigma, Milan, Italy, www.sigmaaldrich.com) $0.10 \% \mathrm{w} / \mathrm{v}$ solution in tap water and portions of the ovary were removed through an incision on the abdomen. The oocytes were treated with collagenase Type IA (Sigma) $1 \mathrm{mg} / \mathrm{mL}$ in calcium-free ND96 for at least $1 \mathrm{~h}$ at $16{ }^{\circ} \mathrm{C}$. After $24 \mathrm{~h}$ at $16{ }^{\circ} \mathrm{C}$ in modified Barth's saline solution (MBS), selected oocytes were injected with $25 \mathrm{ng}$ of cRNA for $\mathrm{sbB}^{0} \mathrm{AT} 1, \mathrm{mB}^{0} \mathrm{AT} 1$ and mCollectrin, and $12,5 \mathrm{ng}$ for msKAAT1 in $50 \mathrm{nl}$ of water, using a manual microinjection system (Drummond Scientific Company, Broomall, PA, www. drummondsci.com). The oocytes were then incubated at $16{ }^{\circ} \mathrm{C}$ for 4-6 days in MBS before electrophysiological studies. The experiments were carried out according to the institutional and national ethical guidelines (permit no. 05/12).

\subsection{Electrophysiology and data analysis}

Measurements of the currents generated by the transporter in controlled voltage conditions were performed using the two-electrode voltage clamp (TEVC) technique (GeneClamp, Molecular Devices, Sunnyvale, CA, USA) (Giovannardi et al., 2007). Intracellular glass microelectrodes, filled with $\mathrm{KCl} 3 \mathrm{M}$ and with tip resistance between 0.5 and $4 \mathrm{M} \Omega$ were used. Agar bridges (3\% agar in $3 \mathrm{M} \mathrm{KCl}$ ) connected the bath electrodes to the experimental chamber. The holding potential $\left(V_{h}\right)$ was $-60 \mathrm{mV}$. Voltage pulses from $-140 \mathrm{mV}$ to $+40 \mathrm{mV}$ in $20 \mathrm{mV}$ increments were applied for $200 \mathrm{~ms}$. Data were analyzed using Clampfit 10.2 (Molecular Devices, www.moleculardevices.com), while statistics and figures were done with Origin 8.0 (originalLab Corp., Northampton, MA, USA, www.originlab.com). Transport currents were obtained by subtracting the current level in the absence of organic substrate from that in its presence. The "leak" currents through the transporter were investigated by comparing the membrane current needed to hold a constant potential in the presence of lithium with respect to sodium, taking as reference the current level in the presence of impermeant tetramethylammonium (TMA ${ }^{+}$) (Bossi et al., 1999b; Hille, 2001).

Pre-steady-state currents were isolated by subtracting the traces in the presence of organic substrate from those in its absence, followed by a further subtraction of the residual steady level of the current at the end of the pulse (Mertl et al., 2008; Bossi et al., 2011). Analysis of the pre-steady-state currents was then performed by calculating the integrals of the isolated transients, to obtain the amount of moved charge $(Q)$, and by fitting the current decay with a single exponential in order to obtain the relaxation time constant $(\tau)$.

\subsection{Solutions}

The oocyte culture and washing solutions had the following composition (in $\mathrm{mM}$ ), calcium-free ND96: $\mathrm{NaCl} 96, \mathrm{KCl} 2, \mathrm{MgCl}_{2}$ 1, HEPES
5, pH 7.6; ND96: $\mathrm{NaCl} 96, \mathrm{KCl} 2, \mathrm{MgCl}_{2}$ 1, $\mathrm{CaCl}_{2}$ 1.8, HEPES 5, pH 7.6; MBS: $\mathrm{NaCl} 88, \mathrm{KCl} 1, \mathrm{NaHCO}_{3} 2.4$, HEPES 15, $\mathrm{Ca}\left(\mathrm{NO}_{3}\right)_{2} 0.30, \mathrm{CaCl}_{2} 0.41$, $\mathrm{MgSO}_{4} 0.82$, sodium penicillin $10 \mu \mathrm{g} / \mathrm{mL}$, streptomycin sulfate $10 \mu \mathrm{g} / \mathrm{mL}$, gentamicin sulfate $100 \mu \mathrm{g} / \mathrm{mL}$, pH 7.6. The external control solution during the electrophysiological recordings had the following composition (mM): $\mathrm{NaCl} 98 ; \mathrm{MgCl}_{2} 1 ; \mathrm{CaCl}_{2} 1.8$, HEPES $5 \mathrm{mM}$, for $\mathrm{pH} 5.5$ solution HEPES was substitute by MES and for the $\mathrm{pH} 8.5$ by TAPS. The $\mathrm{NaCl}$ was totally or partially substituted by $\mathrm{TMACl}, \mathrm{KCl}$ or $\mathrm{LiCl}$ and $\mathrm{Na}$ gluconate in ion dependence experiment. The final pHs were adjusted with $\mathrm{NaOH}$, $\mathrm{KOH}, \mathrm{LiOH}, \mathrm{TMAOH}$ respectively. The substrates, dissolved in water, were added at the indicated concentrations. Experiments were conducted at room temperature $\left(20-25{ }^{\circ} \mathrm{C}\right)$.

\section{Results}

\subsection{Transport currents elicited by different substrates}

In the initial experiments the functional expression of the sea bass $\mathrm{B}^{0} \mathrm{AT} 1$ in the oocytes was investigated by recording the transport currents elicited by various substrates at constant membrane voltage $(-60 \mathrm{mV})$. A representative recording of this kind of current is shown in Fig. 1A, while cumulative data averaged nine oocytes are shown in Fig. 1D. All the neutral amino acids tested elicited inward currents of comparable amplitude when applied at a concentration of $3 \mathrm{mM}$. On the contrary, no currents were generated by the charged amino acid glutamate nor by the dipeptide glycine-glutamine at the same concentration. These results are in substantial agreement with the observations on the renal form of $\mathrm{B}^{0} \mathrm{AT} 1$ from mouse (Camargo et al., 2005), except for the effect of the imino acid proline that appears to generate currents of similar amplitude as leucine in the mouse transporter, but only produces a fast inward transient in the sea bass form. However the fast transient upon proline application can also be seen in non-injected oocytes (Fig. 1C), suggesting that it may be due to endogenous properties of the oocytes. The same sequence of substrates has been applied to another neutral amino acid transporter related to the SLC6A family, KAAT1 from the gut of the invertebrate Manduca sexta (Castagna et al., 1998). The representative record (Fig. 1B) shows distinct differences in comparison to $\mathrm{B}^{0} \mathrm{AT} 1$ (Soragna et al., 2004), see for example the large currents elicited by proline and threonine and the small response to histidine. Finally we tested if the expression was influenced by the accessory protein collectrin as found for the mammalian orthologs, by investigating the effects of co-injection on the current recorded in oocytes expressing $\mathrm{B}^{0} \mathrm{AT} 1$ and KAAT1 (Fig. 1E). As reported (Danilczyk et al., 2006) the transport current was increased five to six-fold in the oocytes co-expressing mouse $\mathrm{B}^{0} \mathrm{AT} 1$ and mouse collectrin. This accessory protein was also able to significantly increase $(p>0.01)$ the activity of $\operatorname{sbB}^{0} \mathrm{AT} 1$, but not that of msKAAT1.

\subsection{Ionic dependence of transport currents}

Previous studies on mammalian $\mathrm{B}^{0} \mathrm{AT} 1$ (Böhmer et al., 2005; Camargo et al., 2005) indicated that, as in the other SLC6A family members, the presence of $\mathrm{Na}^{+}$is essential for transport. As illustrated in Fig. 2, this characteristic is confirmed in $\operatorname{sbB}^{0} \mathrm{AT} 1$. In the total absence of $\mathrm{Na}^{+}$the transport current is abolished if the replacing ion is tetramethylammonium $\left(\mathrm{TMA}^{+}\right.$) or $\mathrm{K}^{+}$, while it is still partially observable when $\mathrm{Na}^{+}$is substituted by $\mathrm{Li}^{+}$(Fig. 2A). We also investigated the possible involvement of $\mathrm{Cl}^{-}$, performing experiments in which this anion was replaced by gluconate: as shown in Fig. $2 \mathrm{~B}$ no significant changes in the $\mathrm{sbB}^{0} \mathrm{AT} 1$ transport current were observed following this substitution, in agreement with the results reported by (Böhmer et al., 2005; O'Mara et al., 2006) on the mouse ortholog, similar results were obtained in the presence of a non saturating dose of substrate (500 $\mu \mathrm{M}$ leucine). 
A

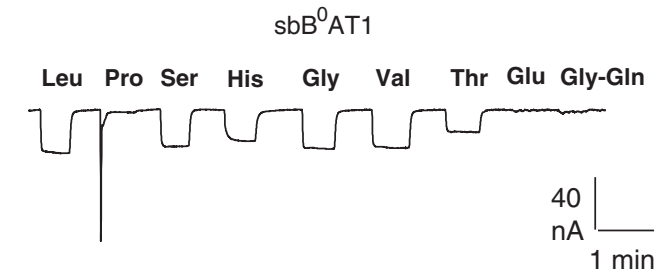

B
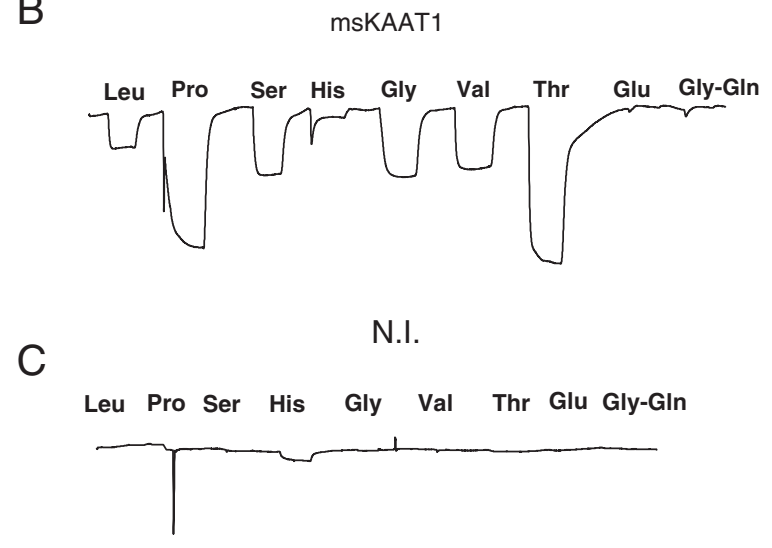

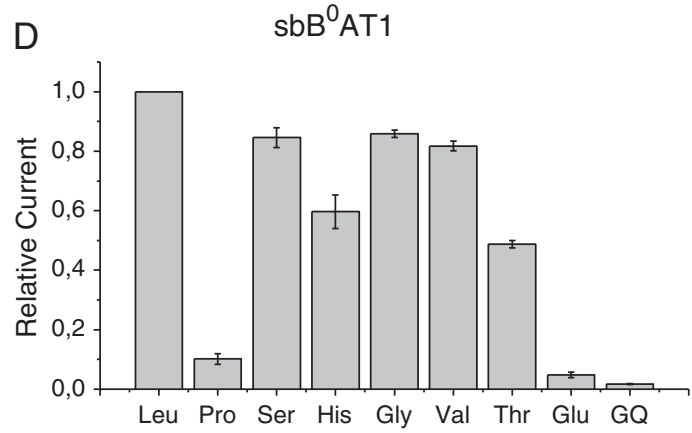

$\mathrm{E}$

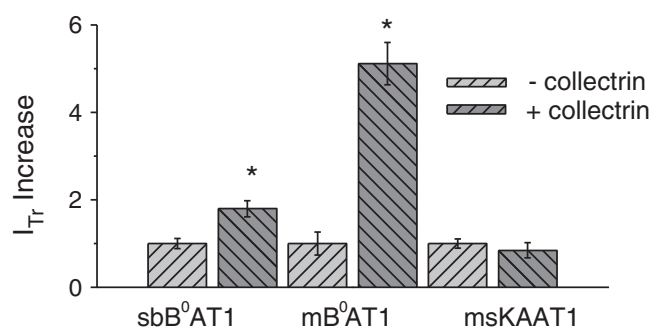

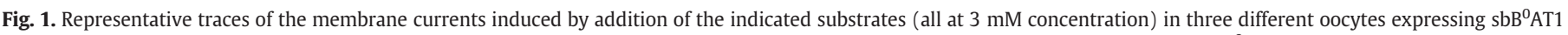

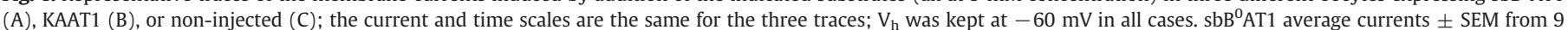

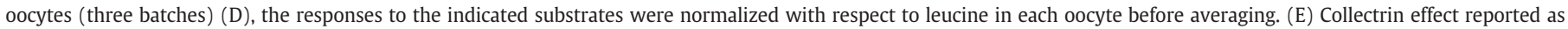

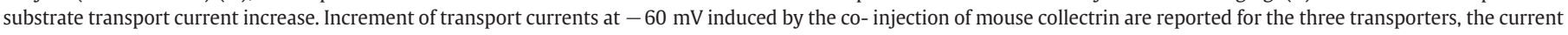

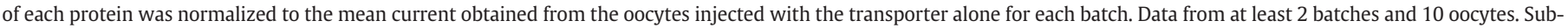
strate were leucine $10 \mathrm{mM}$ for $\mathrm{B}^{0} \mathrm{AT} 1$ and threonine $3 \mathrm{mM}$ for msKAAT1.

\subsection{Apparent affinity}

The apparent affinity for $\mathrm{Na}^{+}$was calculated by fitting a Michaelis-Menten equation to dose-response relationships obtained measuring the transport current elicited at different potentials by $3 \mathrm{mM}$ leucine in the presence of various external $\mathrm{Na}^{+}$concentrations (Fig. $3 \mathrm{~A}$ ). The values of the $\mathrm{Na}^{+}$concentration eliciting half of the maximal current at each potential $\left(K_{0.5}^{\mathrm{Na}}\right)$ are shown in Fig. 3B and are quantitatively and qualitatively in agreement with the results obtained in the mammalian $\mathrm{B}^{0} \mathrm{AT} 1$ (Camargo et al., 2005). The apparent affinity for the organic substrate was similarly obtained for leucine (Fig. 3C). The values of the leucine concentration eliciting half of the maximal current at each potential $\left(K_{0.5}^{\mathrm{Leu}}\right)$ are shown in Fig. 3D and, again, are in good agreement with the results from the mouse $\mathrm{B}^{0} \mathrm{AT} 1$.

\subsection{Effects of $p H$}

We also tested the effects of $\mathrm{pH}$, since the activity of the mouse transporter was shown to be reduced by acidity and slightly increased by alkalinity (Böhmer et al., 2005; Camargo et al., 2005) as for the insect transporter KAAT1 (Peres and Bossi, 2000). Our results are shown in Fig. 4 and qualitatively confirm the previous findings, although quantitatively the effects of $\mathrm{pH}$ on $\mathrm{sbB}^{0} \mathrm{AT} 1$ are smaller, and the effect of acidity appears more like a shift of the curve toward more negative potentials.

\subsection{Leak lithium current}

An interesting distinct feature exhibited by many ion-coupled cotransporters is the existence of transmembrane currents in the
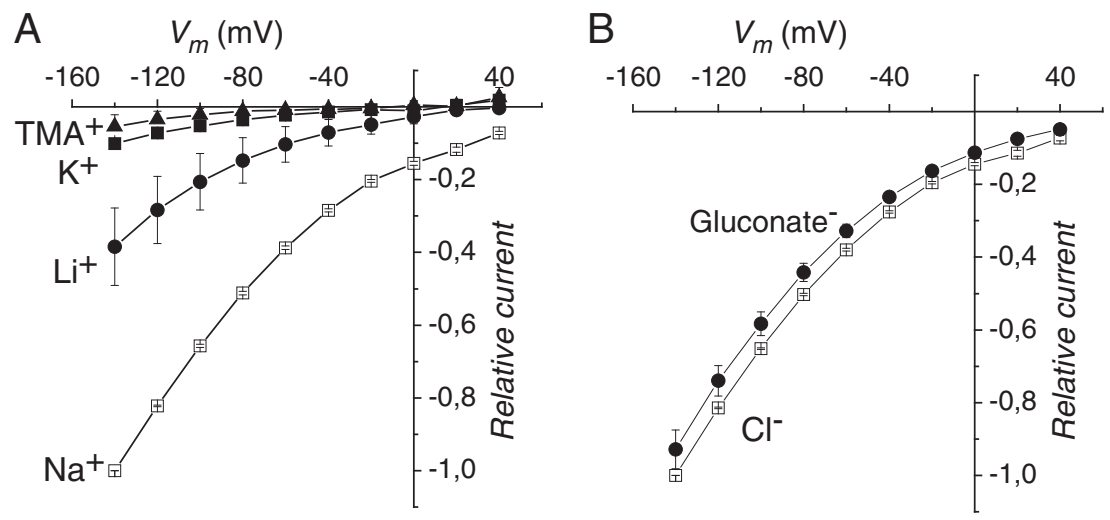

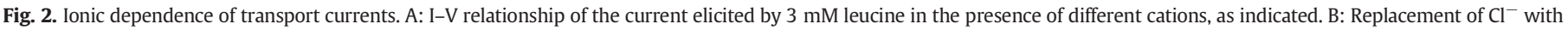

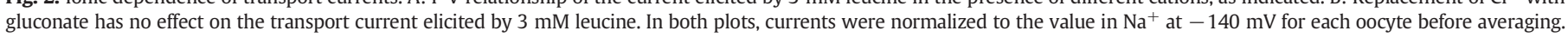
Data are means \pm SEM from 11 oocytes (three batches) in A, and from 10 oocytes (three batches) in B. 

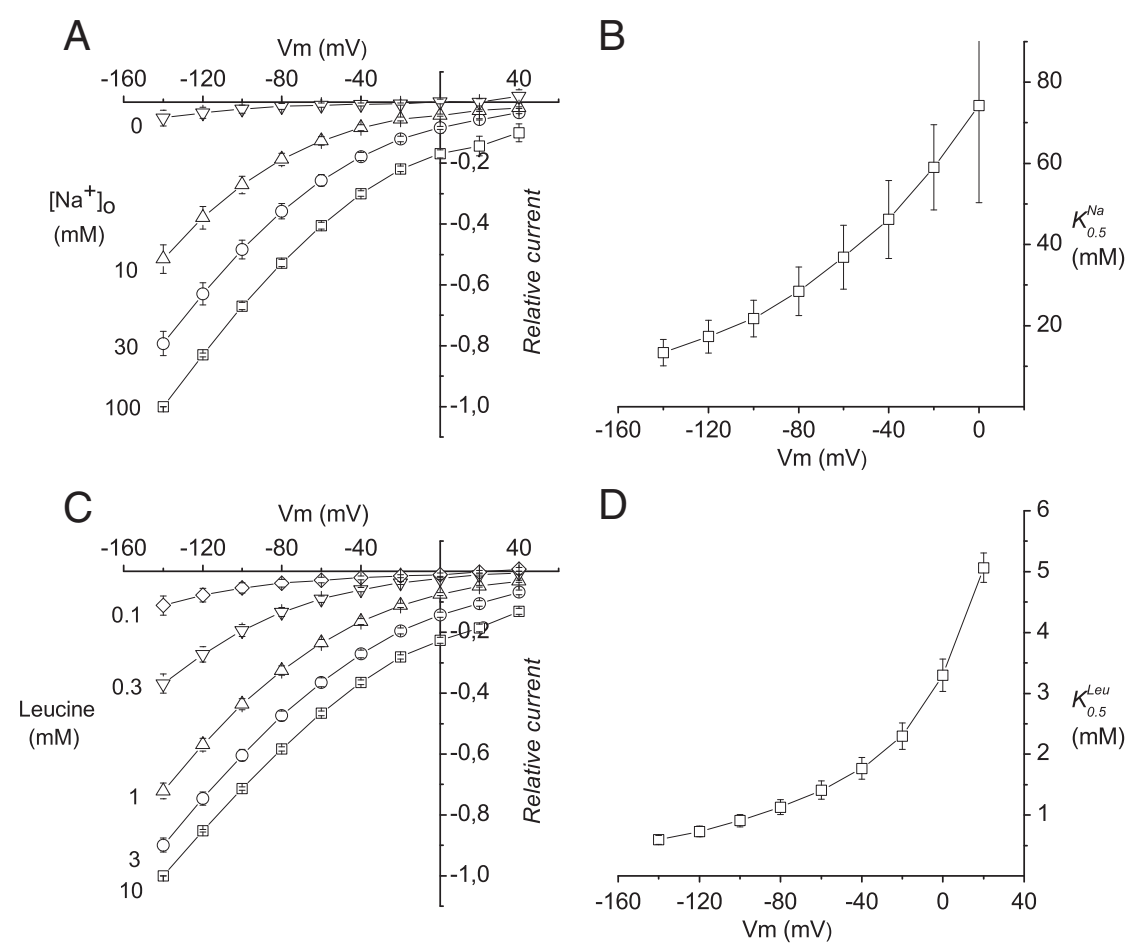

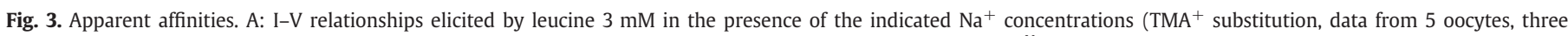

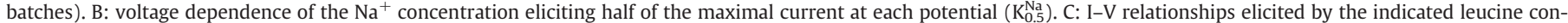

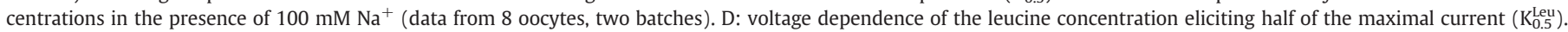

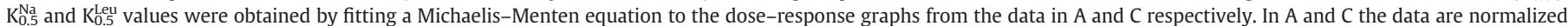
to the current value at $-140 \mathrm{mV}$ in the highest variable substrate concentration.

absence of organic substrate. These "leak" or "slippage" currents (Nelson et al., 2002; Andrini et al., 2008) possess the peculiar characteristic of being best observed when lithium is the permeating ion (Mager et al., 1996; Bossi et al., 1999b; Zhou et al., 2006). In the rappresentative recording of Fig. $5 \mathrm{~A}$ an oocyte expressing $\mathrm{sbB}^{0} \mathrm{AT} 1$ was initially bathed in the control, $\mathrm{Na}^{+}$-containing, solution, giving rise to an holding current of about $-22 \mathrm{nA}$ at $-60 \mathrm{mV}$. As expected, replacement of sodium with $\mathrm{TMA}^{+}$caused a reduction of this current to about zero. When a solution containing lithium as the main cation was perfused on the oocyte, a small inwardly directed current (about $-9 \mathrm{nA}$, definitely smaller than in sodium) developed. This sort of effect is normally seen in oocytes not expressing any transporter, and it is ascribed to the passage of lithium

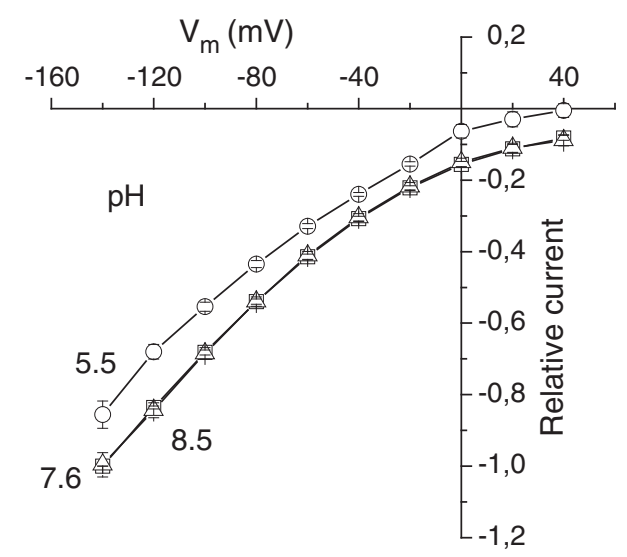

Fig. 4. Effects of $\mathrm{pH}$ on transport currents. The transport currents elicited by $3 \mathrm{mM}$ leucine were measured in the same oocyte at the three indicated $\mathrm{pH}$ values. Currents were normalized at the value at $-140 \mathrm{mV}$ and $\mathrm{pH} 7.6$ (squares) before averaging. Data are means \pm SEM from ten oocytes (three batches). through the endogenous channels of the oocyte (Bossi et al., 1999b). Indeed lithium is normally less permeant than sodium in ionic channels (Hille, 2001). This oocyte was clearly expressing the transporter, as evident from the inward transport current generated by the addition of $3 \mathrm{mM}$ leucine in the presence of sodium in the final part of the record. Therefore this result indicates that $\operatorname{sbB}^{0} \mathrm{AT} 1$ does not show lithium leak currents. As a positive control, we repeated the same kind of experiment on oocytes expressing the insect transporter KAAT1: the representative trace of Fig. 5B shows that in this case the holding current in the presence of a high external lithium solution is much larger than when sodium is present (arrows). This observation, that confirms previous results (Bossi et al., 1999a, 1999b), indicates the existence of a leak pathway in the transporter. We can conclude therefore that $\operatorname{sbB}^{0} \mathrm{AT} 1$ does not offer evidences to be able to carry such kind of current.

\subsection{Pre-steady-state currents}

Generally, the occurrence of pre-steady-state currents in ion-coupled cotransporters can be easily detected during a voltage-clamp experiment as capacitative-like transients with relatively slow decline time constant $(\tau)$ after a voltage step. In most transporters these transient disappear upon addition of the transported substrate, that is, pre-steady-state and transport- associated currents are mutually exclusive (Peres et al., 2002, 2004). The interpretation of these observations is that the intramembrane charge movement causing the pre-steady-state currents represents the initial steps of the transport cycle that can be completed only when the organic substrate is present. The addition of the organic substrate then produces a conversion of the capacitative-like pre-steady-state currents in the resistive-like transport currents (Peres et al., 2002).

In the previous electrophysiological studies on the mouse $\mathrm{B}^{0} \mathrm{AT} 1$ (SLC6A19) (Kleta et al., 2004; Böhmer et al., 2005; Camargo et al., 2005) there is no mention of pre-steady-state currents. Indeed visual inspection of the current traces obtained using voltage steps protocols, 
A

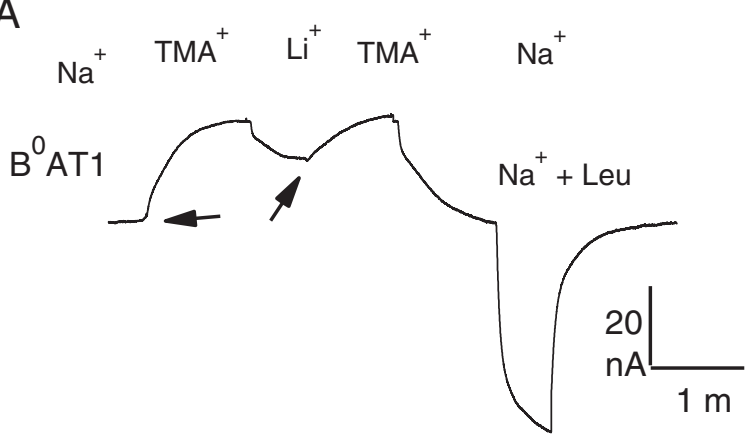

B

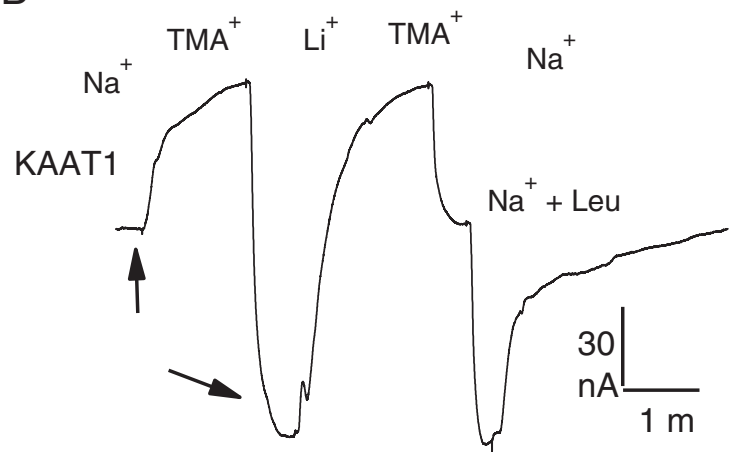

Fig. 5. Absence of lithium-specific leak current in $\operatorname{sbB}^{0} A T 1$. Sample recordings of transmembrane current in two oocytes expressing $\operatorname{sbB}^{0} \mathrm{AT} 1$ (top) or KAAT1 (bottom) at $\mathrm{V}_{\mathrm{h}}=-60 \mathrm{mV}$ and exposed to the indicated external solutions. As in non-injected oocytes, in $\operatorname{sbB}^{0} \mathrm{AT} 1$ the inward current in the presence of $\mathrm{Li}^{+}$is smaller than in $\mathrm{Na}^{+}$(arrows), while in KAAT1 the opposite occurs, indicating the presence of a leak current through the transporter. The response to leucine $(3 \mathrm{mM})$ at the end of the recordings demonstrates that both oocytes were expressing the respective transporters. Analogous results concerning $\operatorname{sbB}^{0} \mathrm{AT} 1$ were observed in three other oocytes from two batches.

such as in Fig. 6, does not reveal the usual slowly-decaying transients in the absence of substrate, nor obvious changes in the time course of the capacitive transients following addition of the organic substrate (Fig. 6A and $\mathrm{B})$. However, when the current traces in saturating substrate concentration (Fig. 6B) are subtracted from the corresponding traces in the absence of substrate (Fig. 6A), the result discloses the existence of very fast transient currents both at the "on" and at the "off" of the voltage pulse (Fig. 6C). These transients may be analyzed as explained in the Method section, allowing the calculation of the amount of charge displaced in the membrane electrical field $(Q)$, and of the time constant of decline $(\tau)$. These data are shown respectively in Fig. 6D and $\mathrm{E}$ as average values from several oocytes. Finally, Fig. $6 \mathrm{~F}$ shows that, as requested for an intramembrane charge movement, the amount of charge moved in the same oocytes when the membrane voltage is changed from -140 to $+40 \mathrm{mV}$ is substantially identical to the amount moved in the opposite direction when the voltage is changed from +40 to $-140 \mathrm{mV}$.

\section{Discussion and conclusion}

\subsection{Comparison with mammalian $B^{0} A T 1$ and KAAT1}

The substrate selectivity of $\mathrm{sbB}^{0} \mathrm{AT} 1$ shows only slight differences with respect to the mouse ortholog previously studied. These are limited to proline that does not elicit significant currents, and to histidine that is transported with an efficiency similar to leucine (while in the mouse shows a $50 \%$ reduced current). The differences in comparison to KAAT1 are more marked, since in the invertebrate transporter proline is actually the best transported amino acid (together with threonine), and histidine is not transported, see Fig. 1 and (Soragna et al., 2004).

Co-expression of mouse collectrin roughly doubled the transport current generated by $\operatorname{sbB}^{0} \mathrm{AT} 1$. This effect is analogous, although definitely smaller, to that observed in the mouse $B^{0} A T 1$, but no effect of collectrin was seen in msKAAT1.

These results encourage further investigations in this direction, although species-specific collectrin is clearly required to obtain more significant effects. However, the expression of $\mathrm{sbB}^{0} \mathrm{AT} 1$ alone was normally quite good, at difference to that of the mouse ortholog, and definitely sufficient to electrophysiologically characterize the transporter.

In general, all these differences might be exploited in future structure-function studies aimed to understand the structural bases of protein interaction, substrate translocation and selectivity.

\subsection{Cationic specificity}

As evident from Fig. 2A, the transport activity is strongly $\mathrm{Na}^{+}$dependent, confirming the findings in the mammalian ortholog (Camargo et al., 2005). However, KAAT1 is known to be able to use also the $\mathrm{K}^{+}$ and, to some extent, the $\mathrm{Li}^{+}$gradient to energize the transport activity (Castagna et al., 1998; Bossi et al., 1999a; Vincenti et al., 2000). Lithium can also partially power up the activity of the mouse $\mathrm{B}^{0} \mathrm{AT} 1$ (Camargo et al., 2005). Our results show that in $\operatorname{sbB}^{0} \mathrm{AT} 1$ no transport current is generated by $\mathrm{K}^{+}$, while a modest activity can be produced by $\mathrm{Li}^{+}$.

It is known that the ability to work with $\mathrm{K}^{+}$represents a functional adaptation of the Manduca sexta intestinal transporters KAAT1 and CAATCH1, because of the peculiar diet of this larva, rich in $\mathrm{K}^{+}$ (Castagna et al., 1998) and it is not a common characteristics of insect NAT transporters (Boudko et al., 2005). An important role in conferring the ability to work with $\mathrm{K}^{+}$in these transporters has been attributed to the presence of an aspartate residue in position 338. In fact, all the other eukaryotic members of the family, unable to use potassium, have an asparagine residue in the corresponding position (Fig. 7). Furthermore, mutation of aspartate 338 to asparagine in KAAT1 was shown to affect the potassium sensitivity in KAAT1 (Mari et al., 2004). The lack of transport activity in $\mathrm{K}^{+}$, exhibited by $\operatorname{sbB}^{0} \mathrm{AT} 1$, may then be related to the presence of an asparagine in position 310 . As noted previously (Mari et al., 2004), it is likely that other residues should be involved as well in determining the cationic selectivity, since for example the bacterial Tyrosine transporter TyT, and other insect NATs have been reported to be unable to work with potassium in spite of the presence of an aspartate in the same position (Boudko et al., 2005; Quick et al., 2006; Meleshkevitch et al., 2009) (Fig. 7).

\subsection{Chloride dependence}

The members of the SLC6A family show different sensitivity to chloride, ranging from strong dependence to no sensitivity (Betté et al., 2008). Substitution of external chloride with gluconate does not seem to significantly affect the transport activity of $\operatorname{sbB}^{0} \mathrm{AT} 1$, as shown by the results of Fig. 2B. Again, KAAT1 is only partially inhibited in the absence of chloride, while contrasting results were reported for the mouse ortholog, from no effect (Böhmer et al., 2005; O'Mara et al., 2006), to partial reduction of the transport current (Camargo et al., 2005).

According to structure-function studies on this matter (Forrest et al., 2007; Zomot et al., 2007), the difference between chloride-sensitive and bacterial homolog, insensitive members of the family is due to the lack, in the former, of intrinsic negative charges in the vicinity of the $\mathrm{Na}^{+}$-binding site(s). Particularly, the presence of two glutamate residues in position 287 and 290 (Fig. 7) in the structure of the SLC6A prototype transporter LeuT (Yamashita et al., 2005) is considered the structural determinant of its chloride-independence. On the other hand, $\mathrm{Cl}^{-}$-dependent transporters, such as GAT1 and SERT1 (Forrest et al., 2007; Zomot et al., 2007) have neutral amino acids in the 
A

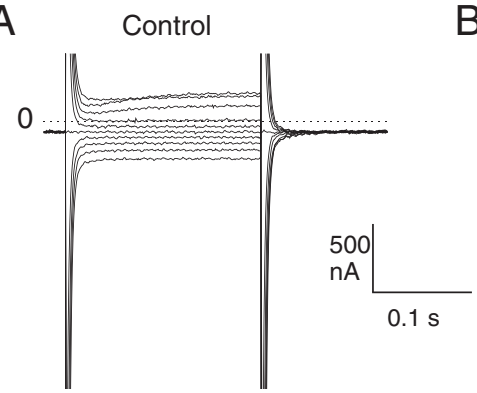

B

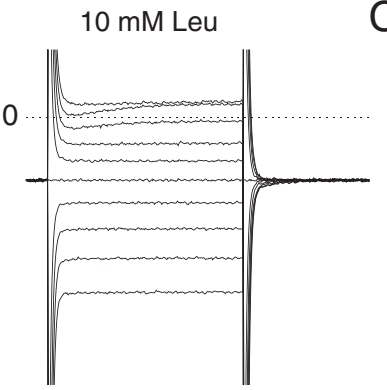

C

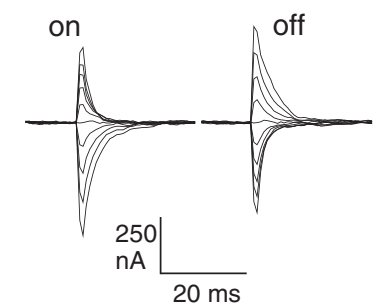

D

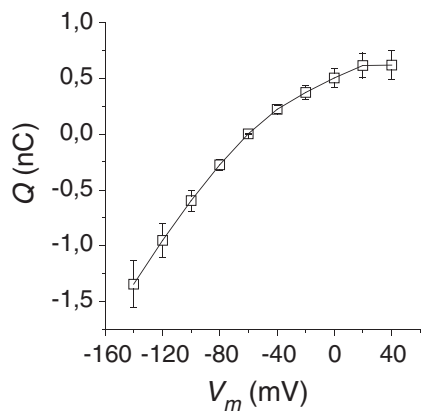

$E$

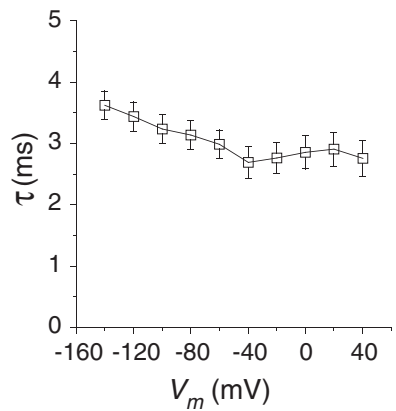

$\mathrm{F}$

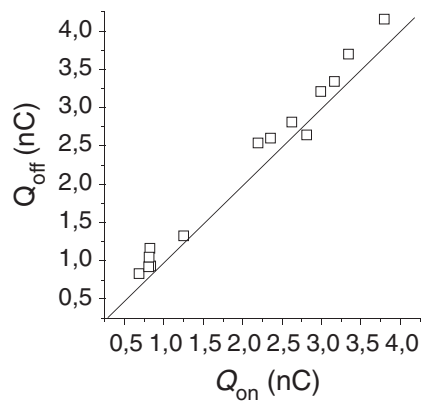

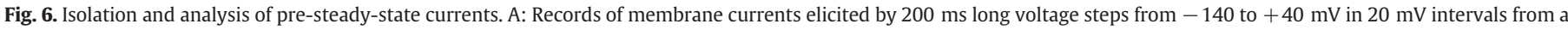

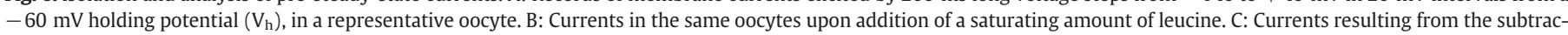

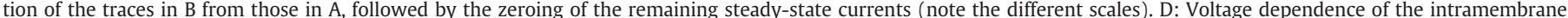

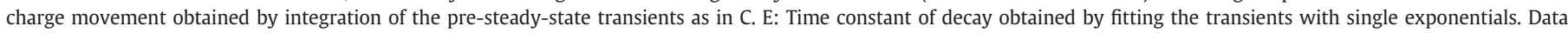

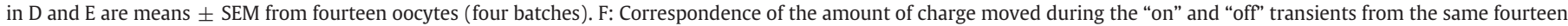
oocytes; the straight line represents equality between $Q_{\text {on }}$ and $Q_{\text {off. }}$

corresponding position, consequently requiring the presence of a mobile negative charge, supplied by a chloride ion, for their correct functioning.

Analysis of the peptide sequence of KAAT1 and $\operatorname{sbB}^{0}$ AT1 shows that the cavity of the $\mathrm{Cl}^{-}$binding site formed by residues such as tyrosine, serine, and asparagine, is conserved in all members of the family. The observed weak dependence of KAAT1 has been attributed to the presence in a nearby position of another negative amino acid (i.e. aspartate 338), also fundamental for the activity in potassium, which could partially contribute the required negative charge; however, $\operatorname{sbB}^{0} \mathrm{AT} 1$ and other NATs transporter (mammalian $\mathrm{B}^{0} \mathrm{AT} 1$ and $\mathrm{B}^{0} \mathrm{AT} 2$, IMINO, and others), that are $\mathrm{Cl}^{-}$-independent, do not appear to have such negative residues in the adjacent regions of the sequence.

No straightforward explanation is therefore available for the virtually chloride-independent behavior of $\operatorname{sbB}^{0} \mathrm{AT} 1$. It is possible, as suggested for the IMINO transporter (Bröer, 2006), that the chloride sensitivity depends on the substrate concentration, becoming minimal at saturation, but this is not the case of $\operatorname{sbB}^{0} \mathrm{AT} 1$, or that these transporters may use a static chloride ion to stabilize their structure (Bröer, 2008).

Yet another hypothesis that could be considered is the indication that in mouse $\mathrm{B}^{0} \mathrm{AT} 1$ the organic substrate may bind first, followed by $\mathrm{Na}^{+}$(Camargo et al., 2005). Commonly, in other transporters, the binding of the organic substrate is believed to occur after the ion interaction (Parent et al., 1992; Peres et al., 2004; Miszner et al., 2007). However, if the opposite order occurs in $\mathrm{sbB}^{0} \mathrm{AT} 1$, it is possible to speculate that the organic substrate may participate in creating the binding pocket for the subsequent binding of $\mathrm{Na}^{+}$, providing perhaps a stabilizing negative charge through its carboxyl end. This possibility would imply that a different mechanism might be adopted by the NAT transporters compared to NTT to return to the outward facing position (Forrest et al., 2007; Zomot et al., 2007).

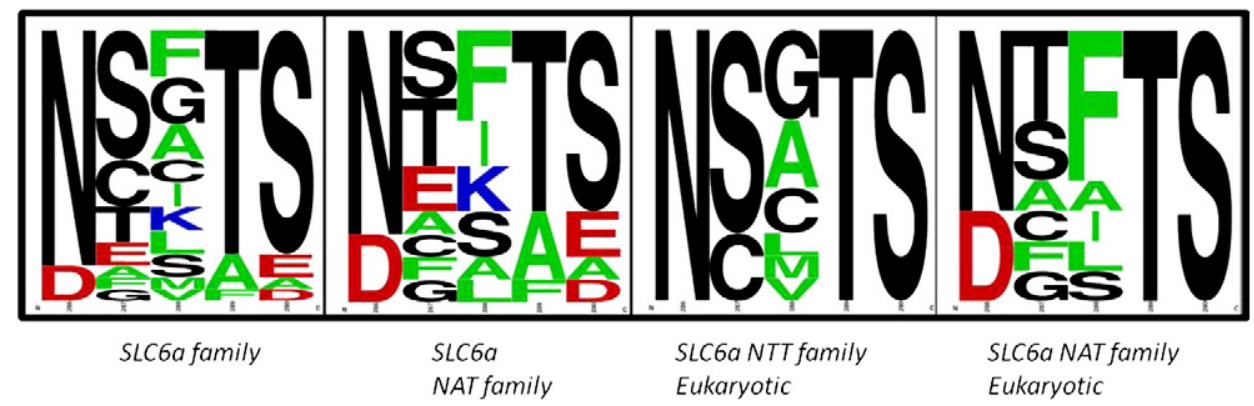

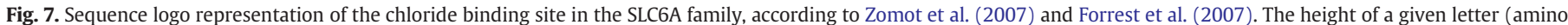

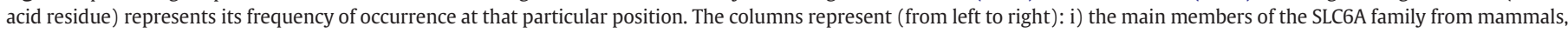

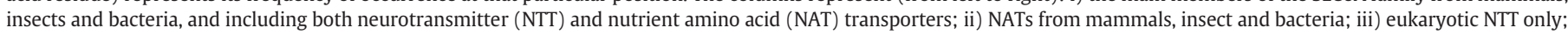
iv) eukaryotic NAT only. 


\subsection{Apparent affinity}

As shown in Fig. 3, the apparent affinities are in the order of 10-60 $\mathrm{mM}$ for $\mathrm{Na}^{+}$and 1-5 $\mathrm{mM}$ for leucine, in good agreement with the values measured in the mouse ortholog (Böhmer et al., 2005; Camargo et al., 2005). Interestingly, the $K_{0.5}$ values for leucine increase at more positive potentials, confirming the observation performed in several other transporter that this parameter is inversely related to the time constant of decline of the pre-steady-state currents (see Fig. 6E).

\section{5. $\mathrm{pH}$ effect}

Differently from the mammalian ortholog, we observed only a modest effect of $\mathrm{pH}$ on the transport activity of $\operatorname{sbB}^{0} \mathrm{AT} 1$. No clear physiological reasons for such difference are apparent, and this observation may be useful for identifying the site of interaction of protons (Camargo et al., 2005). Again, in the SLC6 family different sensitivities to $\mathrm{pH}$ can be found, ranging from strong dependence to a complete insensitivity. Some results suggest an involvement of residue 10.45 (generic numbering (Beuming et al., 2006)) in the pH dependence. A serine residue is present in this position (483) in human and mouse $\mathrm{B}^{0} \mathrm{AT} 1$ while the cloned fish orthologs have instead an alanine (A482). The $\mathrm{pH}$ independent members of NTTs, have in this position a positively charged arginine, but they become $\mathrm{pH}$ sensitive when the arginine is substituted by glutamate in rGAT1 (Forlani et al., 2001) or by threonine in hSERT1 (Cao et al., 1998). It is likely therefore that the non polar alanine in $\operatorname{sbB}^{0} \mathrm{AT} 1$ is responsible for the reduced $\mathrm{pH}$ sensitivity in this ortholog.

\subsection{Transporter leak current}

This transporter property, observed in solute carrier protein in the presence of lithium (Mager et al., 1996; Bossi et al., 1999b; Zhou et al., 2006; Andrini et al., 2008) was not previously investigated in the mouse ortholog. As shown in Fig. 5, our experiments failed to reveal the presence of a leak current in $s b B^{0} A T 1$. In contrast, and as already reported (Bossi et al., 1999a, 1999b) a large lithium-induced current was seen in oocytes expressing the invertebrate KAAT1. It will be interesting to check for the presence of this current in the mouse $\mathrm{B}^{0} \mathrm{AT} 1$ to understand whether this is a difference between vertebrate and invertebrate forms, and possibly to investigate the structural bases of this characteristic. The different order of substrate binding hypothesized for this transporter (see above) might also be relevant to this aspect.

\subsection{Pre-steady-state current}

The $\mathrm{sbB}^{0} \mathrm{AT} 1$ pre-steady-state currents detected in this work are small and fast, and this is the reason why they are not easily detected in the original recordings. The graphs plotted in Fig. 6D and E suggest that the voltage range over which the charge movement occurs is rather wide, extending in the negative direction well beyond the voltages that can be experimentally explored. However, saturation of the $Q-V$ curve occurs at positive potentials, and the $\tau-V$ curve shows a decline that is consistent with right half of a shallow bell-shaped relationship, as expected for the movement of charges between two states (Mager et al., 1996). Finally, the amount of charge moved during the "on" and "off" transitions is equal (Fig. 6F),, These observations satisfy the two properties required for recognizing the intramembrane nature of the charge movement : i) the amount of charge moved by the "on" voltage step must be moved back to the original position by the "off" voltage step (i.e. the integrals of the "on" and "off" current transients must be equal), ii) the voltage dependence of the moved charge should show saturation at extreme positive and negative membrane potentials (Mager et al., 1998).
In conclusion, the electrophysiological characteristics of the transport processes of the sea bass form of $\mathrm{B}^{0} \mathrm{AT} 1$ highlight similarities and differences with respect to the mammalian and insect counterparts. While the analysis of the transport efficiency of the different amino acids and the effects of $\mathrm{pH}$ may be useful for the design of optimal diets in aquaculture, the peculiarities in cationic selectivity and in the effects of chloride might be exploited for structure-function studies, especially in comparison with the orthologs from mammalian and insect species.

\section{Acknowledgements}

This work has been funded under the EU Seventh Framework Programme by the ARRAINA Project $N^{\circ} 288925$ : Advanced Research Initiatives for Nutrition \& Aquaculture. The views expressed in this work are the sole responsibility of the authors and do not necessarily reflect the views of the European Commission.

\section{References}

Andrini, O., Ghezzi, C., Murer, H., Forster, I.C., 2008. The leak mode of type II Na(+)-P(i) cotransporters. Channels 2, 346-357.

Betté, S., Castagna, M., Bossi, E., Peres, A., Sacchi, V.F., 2008. The SLC6/NSS family members KAAT1 and CAATCH1 have a weak chloride dependence. Channels 2, 358-362.

Beuming, T., Shi, L., Javitch, J.A., Weinstein, H., 2006. A comprehensive structure-based alignmentof prokaryotic and eukaryotic neurotransmitter/Na + symporters (NSS) aids in the useof the LeuT structure to probe NSS structure and function. Mol. Pharmacol. 70, 1630-1642.

Böhmer, C., Bröer, A., Munzinger, M., Kowalczuk, S., Rasko, J.E.J., Lang, F., Bröer, S., 2005. Characterization of mouse amino acid transporter B0AT1 (slc6a19). Biochem. J. 389, 745-751.

Bossi, E., Centinaio, E., Castagna, M., Giovannardi, S., Vincenti, S., Sacchi, V.F., Peres, A., 1999a. Ion binding and permeation through the lepidopteran amino acid transporter KAAT1 expressed in Xenopus oocytes. J. Physiol. 515, 729-742.

Bossi, E., Sacchi, V.F., Peres, A., 1999b. Ionic selectivity of the coupled and uncoupled currents carried by the amino acid transporter KAAT1. Pflugers Arch. 438, 788-796.

Bossi, E., Fabbrini, M.S., Ceriotti, A., 2007a. Exogenous protein expression in Xenopus laevis oocyte, Basic procedure. In: Grandi, G. (Ed.), In Vitro Transcription and Translation Protocols. Humana Press, Totowa NJ, pp. 107-131.

Bossi, E., Soragna, A., Miszner, A., Giovannardi, S., Frangione, V., Peres, A., 2007b. Oligomeric structure of the neutral amino acid transporters KAAT1 and CAATCH1. Am. J. Physiol. Cell Physiol. 292, C1379-C1387.

Bossi, E., Renna, M.D., Sangaletti, R., D'Antoni, F., Cherubino, F., Kottra, G., Peres, A., 2011. Residues R282 and D341 act as electrostatic gates in the proton-dependent oligopeptide transporter PepT1. J. Physiol. 589, 495-510.

Boudko, D.Y., 2012. Molecular basis of essential amino acid transport from studies of insect nutrient amino acid transporters of the SLC6 family (NAT-SLC6). J. Insect Physiol. 58, 433-449.

Boudko, D.Y., Kohn, A.B., Meleshkevitch, E.A., Dasher, M.K., Seron, T.J., Stevens, B.R., Harvey, W.R., 2005. Ancestry and progeny of nutrient amino acid transporters. Proc. Natl. Acad. Sci. U. S. A. 102, 1360-1365.

Bröer, S., 2006. The SLC6 orphans are forming a family of amino acid transporters. Neurochem. Int. 48, 559-567.

Bröer, S., 2008. Apical transporters for neutral amino acids: physiology and pathophysiology. Physiology. (Bethesda) 23, 95-103.

Bröer, A., Cavanaugh, J.A., Rasko, J.E.J., Bröer, S., 2005. The molecular basis of neutral aminoacidurias. Pflug Arch 451, 511-517.

Camargo, S.M.R., Makrides, V., Virkki, L.V., Forster, I.C., Verrey, F., 2005. Steady-state kinetic characterization of the mouse $\mathrm{B}^{0} \mathrm{AT} 1$ sodium-dependent neutral amino acid transporter. Pflug. Arch. 451, 338-348.

Camargo, S.M., Singer, D., Makrides, V., Huggel, K., Pos, K.M., Wagner, C.A., Kuba, K., Danilczyk, U., Skovby, F., Kleta, R., Penninger, J.M., Verrey, F., 2008. Tissue-specific amino acid transporter partners ACE2 and collectrin differentially interact with Hartnup mutations. Gastroenterology 136, 872-882.

Cao, Y., Li, M., Mager, S., Lester, H.A., 1998. Amino acid residues that control pH modulation of transport-associated current in mammalian serotonin transporters. J. Neurosci. 18, 7739-7749.

Castagna, M., Shayakul, C., Trotti, D., Sacchi, V.F., Harvey, W.R., Hediger, M.A., 1998. Cloning and characterization of a potassium-coupled amino acid transporter. Proc. Natl. Acad. Sci. U. S. A. 95, 5395-5400.

Daniel, H., Kottra, G., 2004. The proton oligopeptide cotransporter family SLC15 in physiology and pharmacology. Pflug. Arch. 447, 610-618.

Daniel, H., Spanier, B., Kottra, G., Weitz, D., 2006. From Bacteria to Man: Archaic ProtonDependent Peptide Transporters at Work. Physiology 21, 93-102.

Danilczyk, U., Sarao, R., Remy, C., Benabbas, C., Stange, G., Richter, A., Arya, S., Pospisilik, J.A., Singer, D., Camargo, S.M.R., Makrides, V., Ramadan, T., Verrey, F., Wagner, C.A., Penninger, J.M., 2006. Essential role for collectrin in renal amino acid transport. Nature 444, 1088-1091. 
Edwards, H.M., Fernandez, S.R., Baker, D.H., 1999. Maintenance lysine requirement and efficiency of using lysine for accretion of whole-body lysine and protein in young chicks. Poult. Sci. 78, 1412-1417.

Feldman, D.H., Harvey, W.R., Stevens, B.R., 2000. A novel electrogenic amino acid transporter is activated by $\mathrm{K}+$ or $\mathrm{Na}+$, is alkaline $\mathrm{pH}$-dependent, and is $\mathrm{Cl}$-independent. J. Biol. Chem. 275, 24518-24526.

Forlani, G., Bossi, E., Ghirardelli, R., Giovannardi, S., Binda, F., Bonadiman, L., Ielmini, L., Peres, A., 2001. Mutation K448E in the external loop 5 of rGAT1 transporter induces pH sensitivity and altered substrates interactions. J. Physiol. 536, 479-494.

Forrest, L.R., Tavoulari, S., Zhang, Y.-W., Rudnick, G., Honig, B., 2007. Identification of a chloride ion binding site in $\mathrm{Na}+/ \mathrm{Cl}$-dependent transporters. Proc. Natl. Acad. Sci. U. S. A. $104,12761-12766$

Giovannardi, S., Soragna, A., Magagnin, S., Faravelli, L., 2007. Functional expression of type 1 rat GABA transporter in microinjected Xenopus laevis oocytes. 235-255.

Hazama, A., Loo, D.D.F., Wright, E.M., 1997. Presteady-state currents of the rabbit $\mathrm{Na}^{+}$ glucose cotransporter (SGLT1). J. Membr. Biol. 155, 175-186.

Hille, B., 2001. Ionic channels of excitable membranes, 3. Sinauer Ass, Sunderland, MA, USA.

Kleta, R., Romeo, E., Ristic, Z., Ohura, T., Stuart, C., Arcos-Burgos, M., Dave, M.H., Wagner, C.A., Camargo, S.R., Inoue, S., Matsuura, N., Helip-Wooley, A., et al., 2004. Mutations in SLC6A19, encoding B(0)AT1, cause Hartnup disorder. Nat. Genet. 36, 999-1002.

Kwasek, K., Terova, G., Wojno, M., Dabrowski, K., Wick, M., 2012. The effect of dietary dipeptide lysine-glycine on growth, muscle proteins, and intestine PepT1 gene expression in juvenile yellow perch. Rev. Fish Biol. Fish. 22, 797-812.

Mager, S., Kleinberger-Doron, N., Keshet, G.I., Davidson, N., Kanner, B.I., Lester, H.A., 1996. Ion binding and permeation at the GABA transporter GAT1. J. Neurosci. 16, 5405-5414.

Mager, S., Cao, Y., Lester, H.A., 1998. Measurement of transient currents from neurotransmitter transporters expressed in Xenopus oocytes. Methods Enzymol. 296, 551-566.

Margheritis, E., Terova, G., Oyadeyi, A.S., Renna, M.D., Cinquetti, R., Peres, A., Bossi, E., 2012. Characterization of the transport of lysine-containing dipeptides by PepT1 orthologs expressed in Xenopus laevis oocytes. Comp. Biochem. Physiol. A Mol. Integr. Physiol. 164, 520-528.

Mari, S.A., Soragna, A., Castagna, M., Bossi, E., Peres, A., Sacchi, V.F., 2004. Aspartate 338 contributes to the cationic specificity and to the driver-amino acid coupling in the insect cotransporter KAAT1. Cell Mol. Life Sci. 61, 243-256.

Meleshkevitch, E.A., Robinson, M., Popova, L.B., Miller, M.M., Harvey, W.R., Boudko, D.Y., 2009. Cloning and functional expression of the first eukaryotic $\mathrm{Na}+$-tryptophan symporter, AgNAT6. J. Exp. Biol. 212, 1559-1567.

Mertl, M., Daniel, H., Kottra, G., 2008. Substrate-induced changes in the density of peptide transporter PEPT1 expressed in Xenopus oocytes. Am. J. Physiol. Cell Physiol. 295, 1332-1343.

Miller, M.M., Popova, L.B., Meleshkevitch, E.A., Tran, P.V., Boudko, D.Y., 2008. The invertebrate $\mathrm{B}^{0}$ system transporter, D. melanogaster NAT1, has unique D-amino acid affinity and mediates gut and brain functions. Ins. Biochem. Mol. Biol. 38, 923-931.

Miszner, A., Peres, A., Castagna, M., Betté, S., Giovannardi, S., Cherubino, F., Bossi, E., 2007. Structural and functional basis of amino acid specificity in the invertebrate cotransporter KAAT1. J. Physiol. 581, 899-913.
Nelson, N., Sacher, A., Nelson, H., 2002. The significance of molecular slips in transport systems. Nat. Rev. Mol. Cell Biol. 876-881.

O'Mara, M., Oakley, A., Bröer, S., 2006. Mechanism and putative structure of $\mathrm{B}^{0}$-like neutral amino acid transporters. J. Membr. Biol. 213, 111-118.

Parent, L., Supplisson, S., Loo, D.D.F., Wright, E.M., 1992. Electrogenic properties of the cloned $\mathrm{Na}^{+}$/glucose cotransporter: II. A transport model under nonrapid equilibrium conditions. J. Membr. Biol. 125, 63-79.

Peres, A., Bossi, E., 2000. Effects of pH on the uncoupled, coupled and presteady-state currents at the amino acid cotransporter KAAT1 expressed in Xenopus oocytes. J. Physiol. 525, 83-89.

Peres, A., Giovannardi, S., Binda, F., Bossi, E., Fesce, R., 2002. Pre-steady-state and transport-associated currents in the GABA cotransporter rGAT1 are simply related. J. Physiol. 543P, 27P-28P.

Peres, A., Giovannardi, S., Bossi, E., Fesce, R., 2004. Electrophysiological insights on the mechanism of ion-coupled cotransporters. News Physiol. Sci. 19, 80-84.

Quick, M., Yano, H., Goldberg, N.R., Duan, L., Beuming, T., Shi, L., Weinstein, H., Javitch, J.A., 2006. State-dependent conformations of the translocation pathway in the tyrosine transporter Tyt1, a novel neurotransmitter:sodium symporter from Fusobacterium nucleatum. J. Biol. Chem. 281, 26444-26454.

Renna, M.D., Sangaletti, R., Bossi, E., Cherubino, F., Kottra, G., Peres, A., 2011. Unified modeling of the mammalian and fish proton-dependent oligopeptide transporter PepT1. Channels 5, 89-99.

Seow, H.F., Bröer, S., Bröer, A., Bailey, C.G., Potter, S.J., Cavanaugh, J.A., Rasko, J.E.J., 2004. Hartnup disorder is caused by mutations in the gene encoding the neutral amino acid transporter SLC6A19. Nat. Genet. 36, 1003-1007.

Soragna, A., Mari, S., Peres, A., Pisani, R., Castagna, M., Sacchi, V.F., Bossi, E., 2004. Structural domains involved in substrate selectivity in two neutral amino acid transporters. Am. J. Physiol. Cell Physiol. 287, C754-C761.

Terova, G., Bossi, E., Cardillo, A., Harpaz, S., Saroglia, M., 2013. Molecular cloning and characterization of a transporter for neutral amino acids (SLC6A19) in European seabass (Dicentrarchus labrax) reared in freshwater and the effect of different substitutions of fish meal with vegetable meal on its intestinal gene expression levels (in preparation).

Verri, T., Terova, G., Dabrowski, K., Saroglia, M., 2011. Peptide transport and animal growth: the fish paradigm. Biol. Lett. 7, 597-600.

Vincenti, S., Castagna, M., Peres, A., Sacchi, V.F., 2000. Substrate selectivity and pH dependence of KAAT1 expressed in Xenopus laevis oocytes. J. Membr. Biol. 174, 213-224.

Yamashita, A., Singh, S.K., Kawate, T., Gouaux, E., 2005. Crystal structure of a bacterial homologue of $\mathrm{Na}+/ \mathrm{Cl}$-dependent neurotransmitter transporters. Nature 437 215-223.

Zhou, Y., Zomot, E., Kanner, B.I., 2006. Identification of a lithium interaction site in the GABA transporter GAT-1. J. Biol. Chem. 281, 22092-22099.

Zomot, E., Bendahan, A., Quick, M., Zhao, Y., Javitch, J.A., Kanner, B.I., 2007. Mechanism of chloride interaction with neurotransmitter:sodium symporters. Nature 449, 726-731. 